\title{
Application of global optimization to particle identification using light scattering
}

\author{
S Zakovic $†$, Z Ulanowskił and M C Bartholomew-Biggs $†$ \\ $\dagger$ Numerical Optimization Centre, University of Hertfordshire, Hatfield AL10 9AB, UK \\ $\ddagger$ Department of Physical Sciences, University of Hertfordshire, Hatfield AL10 9AB, UK
}

Received 5 November 1997, in final form 22 January 1998

\begin{abstract}
Numerical methods of solving the inverse light scattering problem for spheres are presented. The methods are based on two stochastic global optimization techniques: Deep's random search and the multilevel single-linkage clustering analysis due to Rinnooy Kan and Timmer. Computational examples show that the radius and the refractive index of spheres comparable with or larger than the wavelength of light can be recovered from multiangle scattering data. While the random search approach is faster, the clustering analysis is shown to be more reliable. A general discussion of the clustering method is also given.
\end{abstract}

\section{Introduction}

Substantial advances have been made over the last century or so in the development of the theory of light scattering from small particles. Computation of the properties of scattered electromagnetic fields - the direct scattering problem-is now possible in many situations. Rigorous solutions exist for numerous particle types, such as homogeneous and inhomogeneous spheres, ellipsoids, cylinders, generalized axisymmetric particles and others. Of far greater practical importance, however, is the determination of properties of particles from the knowledge of scattered fields-the inverse scattering problem. Solving it is required in numerous applications, ranging from astronomy and remote sensing, through aerosol and emulsion characterization, to non-destructive analysis of single particles and living cells [1-4].

The inverse problem has proven to be much less amenable to be solved, even for the simplest particle shapes (with the exception of particles much smaller than the wavelength). The lack of rigorous solutions has motivated the development of methods based on approximate models of scattering, for example, assuming that the particles are weak (Rayleigh-Debye) scatterers or that diffraction alone can adequately describe the interaction process. The reader is referred to existing literature for further details [5-7]. However, when such methods are inappropriate, empirical procedures have to be used which are based on generating solutions to the direct problem (after making assumptions concerning the shape, internal structure of the particle, etc) and matching these solutions to experimental data $[2-4,8,9]$. Such procedures can be very slow, difficult to implement and require substantial computing resources. More rapid solutions may be obtainable using neural network methods-an approach to solving inverse problems in multiangle light scattering based on radial basis function (RBF) neural networks, which has recently been proposed, takes advantage of the capability of the RBF networks to approximate multidimensional 
functions [10]. By combining empirical and analytical (eigenfunction) methods, particle size distributions as well as complex refractive indices can be obtained under some constraining conditions [7]. A promising analytical method, which directly yields particle radius but may eventually lead to a full inverse solution, is based on expanding the patterns in terms of a set of Legendre or Gegenbauer polynomials $[11,12]$.

An obvious starting point for attempts to solve the inverse light scattering problem for small particles is the case of a homogeneous, isotropic, non-absorbing sphere. If a plane incident wave of known wavelength and state of polarization and a known medium surrounding the particle are assumed, the particle can be completely described using two parameters, namely its radius $r$ and refractive index $n$. The direct problem can then be solved using the series expansions of the Lorenz-Mie theory [6]. In the scattering geometry considered here, the irradiance of the light scattered by the particle is measured in one plane only. The scattering irradiance can, therefore, be described by a function of the scattering angle (defined as the angle between the direction of the incident wave and the direction of observation). This arrangement leads to a one-dimensional scattering 'pattern' which is representative of the properties of the particle and has been used as a basis for the characterization of both single particles and particle distributions $[1-4,7]$. The present study is confined to such a scattering geometry.

This paper describes the application of global optimization methods, namely the random search method due to Deep [13] and multilevel single-linkage clustering analysis, due to Rinnooy Kan and Timmer [14, 15], to solve an inverse light scattering problem. The problem can be described as follows. If a homogeneous spherical particle is suspended in a known medium and subjected to a plane wave of light then the intensity of the scattered light $I_{1}$ is a function of the scattering angle $\theta$. A theoretical model, called the Lorenz-Mie theory exists (as described in [6]) which gives $I_{1}$ as a function of the radius $r$ and refractive index $n$ of the particle so that $I_{1}(\theta)=\varphi(\theta, r, n)$.

Let us suppose that we have $m$ experimental measures of scattered light intensity

$$
I_{1}\left(\theta_{1}\right), I_{1}\left(\theta_{2}\right), \ldots, I_{1}\left(\theta_{m}\right)
$$

and that we wish to determine corresponding values for $r$ and $n$. A standard approach to such a problem involves finding $r$ and $n$ such that the following function is minimized:

$$
E_{1}=\sum_{i=1}^{m}\left(I_{1}\left(\theta_{i}\right)-k \cdot \varphi\left(\theta_{i}, r, n\right)\right)^{2} .
$$

The additional variable $k$ appears because experimental measurements usually determine relative intensities at the angles $\theta_{i}$ and so a scaling is necessary to provide a match with the theoretical model.

There are a number of optimization techniques which can be used to find the minimum of (1). However, since it is a sum of squared terms, it is appropriate to use the GaussNewton method.

In practice, because the intensities vary widely in magnitude over the typical range $0^{\circ} \leqslant \theta_{i} \leqslant 180^{\circ}$, it may be advisable to consider an objective function of the form

$$
E_{2}=\sum_{i=1}^{m}\left(i\left(\theta_{i}\right)-\psi\left(\theta_{i}, r, n\right)-c\right)^{2}
$$

where $i$ denotes $\log \left(I_{1}\right), \psi$ denotes $\log (\varphi)$ and $c=\log (k)$. Data from experiments are frequently presented in this form in order to give increased weighting to scattering at large angles, which is usually weaker than low-angle scattering. 
The minimization of either $E_{1}$ or $E_{2}$ can be regarded as a three-variable or a two-variable problem. In the case of $E_{1}$ we can write

$$
\partial E_{1} / \partial k=-2 \sum_{i=1}^{m} \varphi\left(\theta_{i}, r, n\right) \cdot\left(I_{1}\left(\theta_{i}\right)-k \cdot \varphi\left(\theta_{i}, r, n\right)\right)
$$

and since $\partial E_{1} / \partial k=0$ at the minimum of (1), we can obtain the optimal value of $k$ in terms of the other two variables:

$$
k=\frac{\sum_{i=1}^{m}\left(I_{1}\left(\theta_{i}\right) \cdot \varphi\left(\theta_{i}, r, n\right)\right)}{\sum_{i=1}^{m} \varphi\left(\theta_{i}, r, n\right)^{2}} .
$$

In a similar way we obtain the optimal value for $c$ in (2):

$$
\begin{aligned}
c & =\frac{\sum_{i=1}^{m}\left(i\left(\theta_{i}\right)-\psi\left(\theta_{i}, r, n\right)\right)}{m} \\
& =\bar{i}-\bar{\psi}
\end{aligned}
$$

where the overbar denotes the mean value over the range $\theta_{1}, \ldots, \theta_{m}$.

It has been shown elsewhere [16-18] that local minimization methods have great difficulties in finding the global optimum of the functions $E_{1}$ and $E_{2}$. The presence of numerous local solutions results in a radius of convergence which is too small for most practical purposes and for this reason it is appropriate to turn to methods of global optimization. In doing so, however, we make the following implicit assumptions. (i) That there exists a unique solution to our inverse light scattering problem. (ii) That this corresponds to the global minimum of $E_{1}$ and $E_{2}$. Mireles [19] has shown that (i) holds for infinite cylinders. However, it is not known whether it is true for homogeneous spherical particles. Nevertheless, the Mireles result, together with other more intuitive reasons, leads to a general consensus in the field of particle characterization that multiangle light scattering data does give unique solutions to the homogeneous sphere problem as long as a sufficient number of measurements is available. In view of assumption (ii) it is, of course, obvious that for 'perfect data' (i.e. data which exactly match the Lorenz-Mie model) the correct solution to the inverse problem will cause both $E_{1}$ and $E_{2}$ to take their global minimum value of zero. However, this remark also highlights the fact that the theoretical question of uniqueness of solutions to the inverse problem may not be very relevant to practical problems of particle identification in which there is the additional complication of accuracy and the discrete character of experimental measurements of scattered light intensity. These complications may arise, for instance, due to finite sampling density and aliasing, incompleteness of data, as well as the presence of noise. Further discussion of these issues is available elsewhere $[10,18,20]$. Within the scope of this paper we simply take it as a convenient and reasonable assumption that global optimization of $E_{1}$ or $E_{2}$ will yield to correct identification of a particle from its light-scattering pattern.

Two global optimization approaches which have proved successful in many applications are described in the next section. Further, in this paper we investigate the use of these methods for solving the inverse scattering problem for perfect data, i.e. the scattered intensities $I_{1}$ computed from the Lorenz-Mie theory, for homogeneous spheres comparable in size with the wavelength of light, corresponding to size parameter $x$ between 13 and 73 ( $x=2 \pi n_{0} r / \lambda$, where $n_{0}$ is the refractive index of the medium and $\lambda$ is the wavelength in the vacuum). 


\section{Stochastic global optimization methods}

All stochastic algorithms break down into two phases: a global and a local one. In the global phase the objective function is evaluated at a number of randomly sampled points drawn from a distribution over a domain $S$; then, in the local phase, sample points obtained from the global phase are manipulated usually by means of a local search procedure. The efficiency of a global optimization method depends greatly on the way in which the set of sample points is updated. A purely deterministic multistart strategy which performs many local optimizations from a large number of starting points can be very wasteful. Several local searches started from many different points may all lead to the same local minimum and a good local phase should detect this situation and prevent the waste of effort involved in finding the same solution more than once.

The final stage of a stochastic algorithm is to decide when to stop searching for any further candidate solutions for the global minimum. A stopping rule must make some prediction about the probability that the function minimum has been located after each iteration. Once the iteration is stopped, the candidate solution with the smallest function value is declared the global minimum.

\subsection{Deep's method}

This method seeks the global solution to the following problem:

$$
\min _{x} f(x) \quad x \in S \subseteq R^{q}
$$

where $S$ is defined by

$$
\begin{array}{lc}
g_{j}(x) \geqslant 0 & j=1,2, \ldots, m \\
\underline{x_{i}} \leqslant x \leqslant \overline{x_{i}} & i=1,2, \ldots, q .
\end{array}
$$

This algorithm has been applied to a wide range of optimization problems arising in the field of science and engineering [21]. It is very easy to use because it does not require any continuity and differentiability conditions of the functions involved and because, unlike a local minimization method, its performance does not depend critically on a single initial guessed value for the unknowns.

The steps of the algorithm are as follows.

(1) Determine the initial array of $N=10(q+1)$ random feasible points and store them and their function values in an $N \times(q+1)$ array $A$. Set ITER $=$ ITER +1 .

(2) Determine $L$ and $M$, the points with the least and greatest function values $f(L)$ and $f(M)$. If

$$
\left|\frac{f(M)-f(L)}{f(M)}\right| \leqslant \epsilon
$$

then $L$ is the point of the global minimum, stop. Otherwise set ITER $=$ ITER +1 and go to step (3).

(3) Determine $N-1$ trial points $P_{1}, P_{2}, \ldots, P_{N-1}$, where each $P_{i}$ is the minimum of the quadratic curve passing through $R_{1}, R_{2}$ and $R_{3}$, where $R_{1}=L$, and $R_{2}$ and $R_{3}$ are randomly selected from the points of the array $A$. If $f\left(P_{i}\right)<f(M)$ then replace $M$ by $P_{i}$ in the array $A$ and go to (2). If either $P_{i}$ is not feasible or $f\left(P_{i}\right) \geqslant f(M)$ then new values for $R_{2}$ and $R_{3}$ are determined.

(This algorithm does not truly find each $P_{i}$ as a local minimum, but the quadratic curve search can be regarded as a 'local phase'.) The algorithm mentioned here still lacks 
efficiency because the same local minimum may be found several times. If we define the region of attraction of a local minimum $x^{*}$ to be the set of points in $S$ starting from which a given local search procedure converges to $x^{*}$, then ideally this local search procedure should be started exactly once in every region of attraction. Methods based on this principle are known as clustering methods.

\subsection{Clustering method}

In this section a stochastic method due to Rinnooy Kan and Timmer $[14,15]$ is described. We wish to minimize a twice differentiable objective function $f: S \rightarrow R$, within the region defined by the $q$-dimensional box $S$.

The method combines the ideas of random search and multistart techniques. On every iteration new random sample points are selected in the box $S$. Instead of simply evaluating the objective function at these points, local optimizations are started from some of them, depending on certain criteria. The local minima that are found are then stored in a list and, when the algorithm terminates, the local minimum with the smallest value of the objective function is declared to be the global minimum.

The multilevel single-linkage algorithm uses the function values at each sample point in deciding which of them will be a start for a local search. In particular, no search is started from a point $x$ whose function value exceeds a specified threshold. Furthermore, for any sample point $x$ where $f(x)$ is sufficiently small, the decision to start a local minimization also depends on whether another point $z$, such that $f(x)<f(z)$, is within a certain distance of $x$. This critical distance is defined at iteration $j$ as

$$
r_{j}=\pi^{-1 / 2}\left(\Gamma\left(1+\frac{q}{2}\right) m_{L}(S) \frac{\sigma \log j N}{j N}\right)^{1 / q}
$$

where $\Gamma(\cdot)$ denotes the gamma function, $m_{L}(\cdot)$ denotes the Lebesgue measure and $\sigma>0$ is an arbitrarily chosen constant (Dixon and Jha, for example, use $\sigma=1.9$ in [22] whereas Rinnooy Kan and Timmer use $\sigma=4$ in [15]). As a result of these factors, a random sample of $N$ points gives rise to a reduced sample of $\gamma N$ points, $\gamma<1$. It is the reduced sample which contains starting points for a set of local optimizations.

A group of points which are within a critical distance $r_{j}$ of each other is called a cluster; and the process of including only one point from any cluster in the reduced sample is called clustering. During the optimization, the critical distance $r_{j}$ decreases, and since any two local minima will always be separated by a region with higher values, multilevel single linkage will locate every local minimum in a neighbourhood of which a point has been sampled.

Since the method is probabilistic in nature it cannot be guaranteed, at any stage, that all local minima in the region of interest have been found. However, a Bayesian estimation of the expected number of minima observed after a certain number of local optimizations can be used. Let $N$ be the number of local optimizations performed, and $W$ be the number of local optima found. Then, the posterior expectation of the number of local minima is:

$$
W_{\mathrm{tot}}=\frac{W(N-1)}{N-W-2} .
$$

Although the true number of local minima is an integer, and (7) will yield a non-integer estimate, it can be verified that the optimal integer Bayesian estimate is a round-off of the non-integer estimate [23]. Therefore, after the $j$ th iteration the algorithm is terminated if

$$
W_{\text {tot }}<W+0.5 \text {. }
$$


The algorithm is as follows (at iteration $j$ ).

- Step 1. Generate $N$ more sample points from a uniform distribution over $S$.

- Step 2. Reduce the sample to $\gamma j N$ points.

- Step 3. Calculate $r_{j}$, using (6).

- Step 4. Order the sample points so that $f\left(x_{i}\right) \leqslant f\left(x_{i+1}\right), i=1,2, \ldots, \gamma k N-1$. For each value of $i$, start local minimization from $x_{i}$, unless there is a sample point $x_{k}$ with $f\left(x_{k}\right)<f\left(x_{i}\right)$ and $\left\|x_{k}-x_{i}\right\| \leqslant r_{j}$, and add the result to the cluster if it is not a known local minimum.

- Step 5. Test the Bayesian stopping rule and, if necessary, go to step 1 with $j$ incremented by 1 .

\section{Computational results}

In this section typical results are given, obtained using the two global methods described above in conjunction with the Gauss-Newton subroutine opls from the OPTIMA library [24] as the local minimizer for the objective functions. Computations of the scattered intensity functions $I_{1}$ and $\varphi$ were carried out using the Lorenz-Mie theory [6]. The range of scattering angles was $0^{\circ} \leqslant \theta_{i} \leqslant 180^{\circ}$ and the scattering functions were sampled with $1^{\circ}$ increments. A wavelength of $0.5145 \mu \mathrm{m}$, incident light polarized perpendicularly to the scattering plane and a refractive index of the surrounding medium (water) of 1.336 were assumed. Gradients of $\varphi$ with respect to $r$ and $n$ (in the clustering approach) were obtained using automatic differentiation [16-18].

\subsection{Using Deep's random search method}

This method requires a seed number for a random number generator. In the tests that follow 40 different seeds were used, meaning that 40 independent runs were performed for each of the tests. The bounds introduced to define the box $S$ were $1.3 \leqslant n \leqslant 1.7$ and $1 \leqslant r \leqslant 2$, with $n$ representing the refractive index and $r$ the radius in micrometres. The test problems considered had a global solution with $n^{*}=1.525$ and $r^{*}=1.475 \mu \mathrm{m}$ (size parameter of about 24).

Table 1 shows the results of the optimization of the function (1) as a two-variable problem, where $k$ is determined from (4). It can be seen that even this method, which is intended to be a global one, can encounter local minima. In four cases, two of which were

Table 1. Results of minimizing (1) with respect to $n$ and $r$ with 30 and 90 initial points (IP). The number of times each minimum was found (out of the total of 40) and the smallest and largest number of iterations for each minimum are given.

\begin{tabular}{|c|c|c|c|c|}
\hline \multirow[b]{2}{*}{ IP } & \multicolumn{2}{|c|}{ Point found } & \multirow[b]{2}{*}{ Hits } & \multirow[b]{2}{*}{ Iterations } \\
\hline & Ref. index & Radius $(\mu \mathrm{m})$ & & \\
\hline 30 & 1.525 & 1.475 & 31 & $239-683$ \\
\hline 30 & 1.657 & 1.682 & 1 & 182 \\
\hline 30 & 1.679 & 1.615 & 2 & $241-248$ \\
\hline 30 & 1.695 & 1.557 & 2 & $168-468$ \\
\hline 90 & 1.525 & 1.475 & 31 & 598-929 \\
\hline 90 & 1.695 & 1.558 & 2 & $444-500$ \\
\hline
\end{tabular}


Table 2. Results of minimizing (1) with respect to $n, r$ and $k$ with 40 initial points.

\begin{tabular}{|c|c|c|c|c|}
\hline \multirow[b]{2}{*}{ No } & \multicolumn{2}{|c|}{ Point found } & \multirow[b]{2}{*}{$k$} & \multirow[b]{2}{*}{ Iterations } \\
\hline & Ref. index & Radius $(\mu \mathrm{m})$ & & \\
\hline 1 & 1.658 & 1.681 & 0.591 & 356 \\
\hline 2 & 1.525 & 1.475 & 0.999 & $20258^{\mathrm{a}}$ \\
\hline 3 & 1.538 & 1.433 & 1.274 & $517^{\mathrm{b}}$ \\
\hline 4 & 1.525 & 1.475 & 1.000 & $12080^{\mathrm{a}}$ \\
\hline 5 & 1.525 & 1.475 & 0.999 & $14125^{\mathrm{a}}$ \\
\hline
\end{tabular}

${ }^{a}$ Failures of the algorithm.

${ }^{b}$ A point which is not a solution.

very near the global solution, the algorithm failed (these failures are discussed below). All the minima found in table 1 were genuine (i.e. $\nabla E_{1}=0$ ).

One of the reasons for finding a local solution instead of the global one could be that the initial number of points where the function is computed is quite small $\left(10\left(n_{q}+1\right)\right.$, where $n_{q}$, as before, represents the number of variables). This may mean that, especially when a large search domain is involved, the function is not evaluated at enough points, allowing the global solution to be missed. To overcome this difficulty, the number of points at which the function is initially evaluated was increased three-fold in the next test, the results of which are also given in table 1. Now only two minima are found, compared with four previously. However, the number of iterations increased considerably (the maximum number of iterations was 929, compared with 683). Also, seven failures of the algorithm can be noted, whereas previously there were four.

In the next example, optimization of function (1) is treated as a three-variable problem. The refractive index and radius boundaries remain the same and bounds of $0.5 \leqslant k \leqslant 2.0$ for $k$ are introduced, as $k$ is expected to be 1 at the global solution or near 1 at a local solution. It turns out that the problem with three variables is much more difficult to solve, therefore only five runs were done, instead of 40 as previously. However, this is enough to show difficulties with the algorithm. The results are shown in table 2. It can be seen that the algorithm failed four times out of 5, and that the number of iterations has increased enormously in some cases. The algorithm had difficulties with terminating properly and failed very close to the global solution in three cases. One local and the global solution were found, whereas in run 3 it terminated at a point which was not a solution at all.

To understand the failures we must look at step 3 in Deep's algorithm, which was fully described in section 2.1 .

$L$ and $M$ are the points with the least and greatest function values $f(L)$ and $f(M)$. It can be seen that if either a feasible point or a point with a smaller function value than the current $f(M)$ could not be found the algorithm would continue trying to find such points. In cases where $f(M)$ is very close to a local solution, it is possible that none of the points from the array $A$ satisfy those criteria, forcing the algorithm to cycle. After a certain number of iterations the algorithm would terminate, reporting the error message that a multiple search has been encountered.

In previous studies where the performance of the two models (1) and (2) was compared, it was concluded that both models produced similar results and had similar radius of convergence and number of local minima $[16,17]$. In the following example Deep's algorithm is tested using the error function (2). Initially, (2) is treated as a two-variable problem. The same boundaries for $r$ and $n$ are used as before and the number of initial 
Table 3. Results of minimizing (2) with respect to $n, r$ and $c$ with $10\left(n_{q}+1\right)$ initial points.

\begin{tabular}{llllll}
\hline \multicolumn{3}{c}{ Point found } & & & \\
\cline { 1 - 2 } Ref. index & Radius $(\mu \mathrm{m})$ & $c$ & & Hits & Iterations \\
\hline 1.525 & 1.475 & - & 40 & $132-210$ \\
1.525 & 1.475 & 0.000 & 39 & $253-533$ \\
1.519 & 1.387 & -0.059 & 1 & 339 \\
\hline
\end{tabular}

Table 4. Results of minimizing (2) with respect to $n$ and $r$ for data set 1 .

\begin{tabular}{lllr}
\hline & Ref. index & Radius $(\mu \mathrm{m})$ & \multicolumn{1}{l}{ Error } \\
\hline 1 & 1.52500 & 1.47500 & 0.00000 \\
2 & 1.51872 & 1.38713 & 26.49644 \\
3 & 1.51966 & 1.53426 & 27.78892 \\
4 & 1.51659 & 1.56796 & 28.00828 \\
5 & 1.52387 & 1.39881 & 28.64589 \\
6 & 1.52629 & 1.56261 & 28.87988 \\
\multicolumn{4}{c}{ Total number of local optimizations: 108} \\
\hline
\end{tabular}

points at which the error function is evaluated is $10\left(n_{q}+1\right)$. The results are shown in table 3. The situation with the logarithmic data is clearly better. Convergence was achieved for all 40 runs and the algorithm did not fail.

Whereas the algorithm had difficulty in terminating during optimization as the threevariable problem with the linear data, the situation with logarithmic data is improved, as can be seen from table 3 . The bounds on $c$ are $-0.5 \leqslant c \leqslant 0.5$. There were 39 convergences to the global and one to a local solution. The number of iterations varied from 253 to 533 , which is twice that for the two-variable problem. As in the previous case, no multiple searches were encountered.

\subsection{Using the clustering approach}

In the first test, the clustering algorithm is applied to the two-variable form of (2) in the region which contains all the local minima encountered in previous studies $[16,17]$. The box is $1.475 \leqslant n \leqslant 1.575$ and $1.375 \leqslant r \leqslant 1.575 \mu \mathrm{m}$ and the initial sample size is 100 points. The results are shown in table 4 . It can be seen that six minima were found, including the global one (it has zero error).

The same algorithm is now applied to the three-variable problem (table 5). The box for refractive index and radius remains the same and the bounds on the scaling factor are $c(-0.5 \leqslant c \leqslant 0.5)$. Performance is almost the same as for the two variable problem. For this number of local minima the algorithm had no difficulties in finding all of them.

The next example (data set 2) is based on scattering data for a spherical particle with refractive index $=1.4$ and radius $=1.0 \mu \mathrm{m}$. The problem is in its two variable form, and the boundaries for refractive index and radius are $1.3 \leqslant n \leqslant 1.5$ and $0.85 \leqslant r \leqslant 1.15 \mu \mathrm{m}$. The results shown in table 6 are based on an initial sample of 100 starting points. The outcome is better than previously-despite a bigger box, fewer minima were found.

The behaviour of the error function is illustrated in figure 1. It shows $-E_{2}$ so that 
Table 5. Results of minimizing (2) with respect to $n, r$ and $c$ using data set 1 .

\begin{tabular}{lllrr}
\hline & Ref. index & Radius $(\mu \mathrm{m})$ & \multicolumn{1}{l}{$c$} & \multicolumn{1}{l}{ Error } \\
\hline 1 & 1.52500 & 1.47500 & 0.00000 & 0.00000 \\
2 & 1.51872 & 1.38713 & -0.05879 & 26.49644 \\
3 & 1.51966 & 1.53426 & 0.01946 & 27.78892 \\
4 & 1.51659 & 1.56795 & 0.03631 & 28.00828 \\
5 & 1.52387 & 1.39881 & -0.06165 & 28.64589 \\
6 & 1.52629 & 1.56260 & 0.08311 & 28.87988 \\
\multicolumn{5}{c}{ Total number of local optimizations: } \\
\hline
\end{tabular}

Table 6. Results of minimizing (2) with respect to $n$ and $r$ using data set 2 .

\begin{tabular}{lllr}
\hline & Ref. index & Radius $(\mu \mathrm{m})$ & \multicolumn{1}{l}{ Error } \\
\hline 1 & 1.40000 & 1.00000 & 0.00000 \\
2 & 1.41834 & 1.08951 & 37.76969 \\
3 & 1.48204 & 0.92848 & 52.47595 \\
4 & 1.49066 & 1.10542 & 60.82964 \\
\multicolumn{3}{c}{ Total number of local optimizations: 51} \\
\hline
\end{tabular}

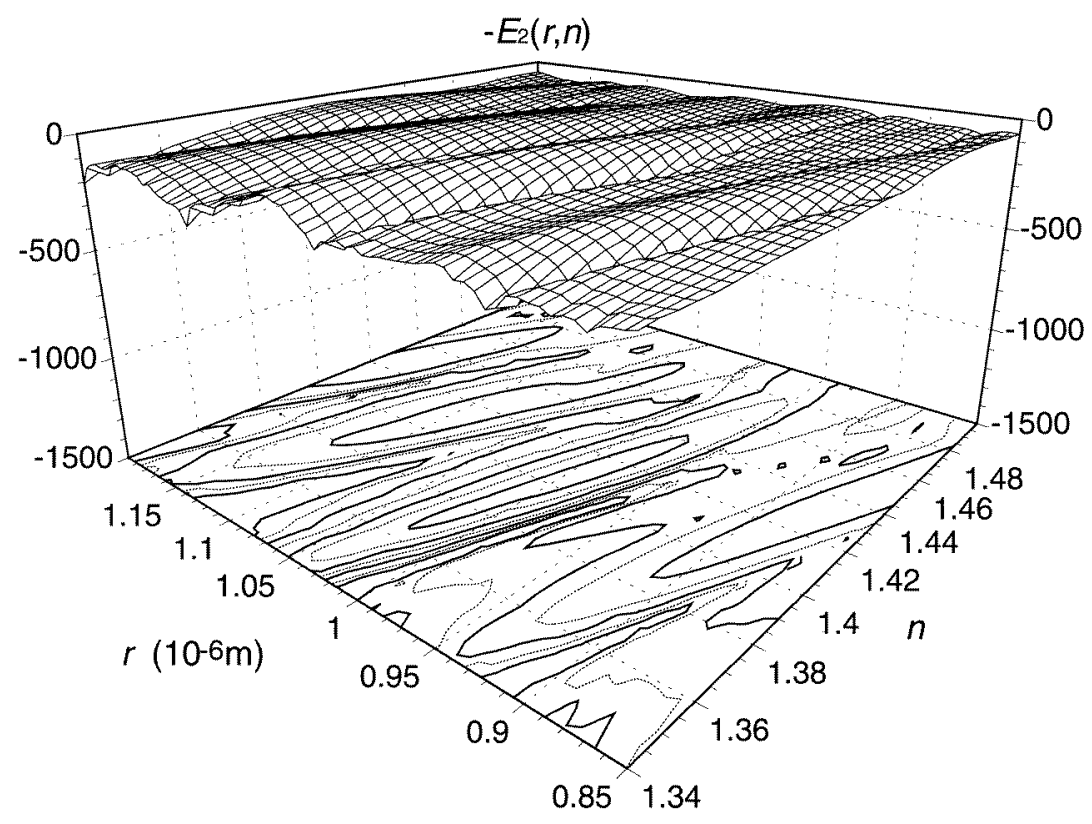

Figure 1. The error surface $-E_{2}(r, n)$ for data set 2. The global solution is at $r^{*}=1.00 \mu \mathrm{m}$, $n^{*}=1.40$.

minima of $E_{2}$ are more clearly visible. Also, this graph appears to show the presence of more than four minima. To check whether there were more minima, the starting sample size was increased from 100 to 500, so the starting points were more dense, and the chance of missing a local minimum was smaller. The results are shown in table 7 and there are 
Table 7. Results of minimizing (2) with respect to $n$ and $r$ using data set 2 with 500 starting points.

\begin{tabular}{lllr}
\hline & Ref. index & Radius $(\mu \mathrm{m})$ & \multicolumn{1}{l}{ Error } \\
\hline 1 & 1.40000 & 1.00000 & 0.00000 \\
2 & 1.41834 & 1.08951 & 37.76969 \\
3 & 1.48204 & 0.92848 & 52.47595 \\
4 & 1.49066 & 1.10542 & 60.82964 \\
5 & 1.48798 & 0.89420 & 68.42821 \\
6 & 1.43730 & 0.90879 & 82.26707 \\
7 & 1.41486 & 0.93911 & 82.89519 \\
\multicolumn{4}{c}{ Total number of local optimizations: 144} \\
\hline
\end{tabular}

Table 8. Results of minimizing (2) with respect to $n$ and $r$ in boxes of size $0.02 \times 0.15$.

\begin{tabular}{rllcc}
\hline \multicolumn{3}{c}{ global solution } & & \\
\cline { 2 - 3 } & Ref. index & Radius $(\mu \mathrm{m})$ & Iterations & Minima \\
\hline 1 & 1.350 & 0.800 & 20 & 2 \\
2 & 1.350 & 3.500 & 88 & 5 \\
3 & 1.375 & 1.500 & 18 & 2 \\
4 & 1.375 & 3.000 & 25 & 2 \\
5 & 1.400 & 2.000 & 42 & 3 \\
6 & 1.400 & 4.000 & 32 & 3 \\
7 & 1.500 & 0.800 & 12 & 1 \\
8 & 1.500 & 2.000 & 21 & 2 \\
9 & 1.500 & 3.750 & 18 & 2 \\
10 & 1.550 & 2.500 & 126 & 7 \\
11 & 1.550 & 3.000 & 303 & 11 \\
12 & 1.550 & 4.500 & 726 & 18 \\
13 & 1.600 & 1.000 & 23 & 2 \\
14 & 1.600 & 4.500 & 123 & 7 \\
15 & 1.650 & 2.500 & 162 & 8 \\
\hline
\end{tabular}

now seven local minima.

It is worth noting that the minima that are missing from table 6 are those with bigger error values (minima numbers 5, 6 and 7). This is due to the fact that the algorithm uses a reduced sample, which disregards certain points with higher function values. This is a useful feature, since the main aim in practice is not to find all local minima, but only those with smaller function values. For the purpose of this section, where we are interested in the behaviour of the error function, the bigger initial sample of 500 points will be used. (Another test was made with 1000 initial points, and obtained the same results as in table 7; therefore, 500 seems a suitable sample size to use.)

In order to find out whether $E_{2}$ has different numbers of local minima when applied to data involving different regions of the $(r, n)$ plane, global solutions based on values of $r$ and $n$ randomly chosen from the area of interest are now considered. Table 8 shows the number of minima found for 15 different data sets obtained by running the Lorenz-Mie model with some nominal values $n^{*}$ and $r^{*}$. The region used in each case is centred on the nominal global solution point $\left(n^{*}, r^{*}\right)$ which represents the idealized particle used to 
Number of local minima

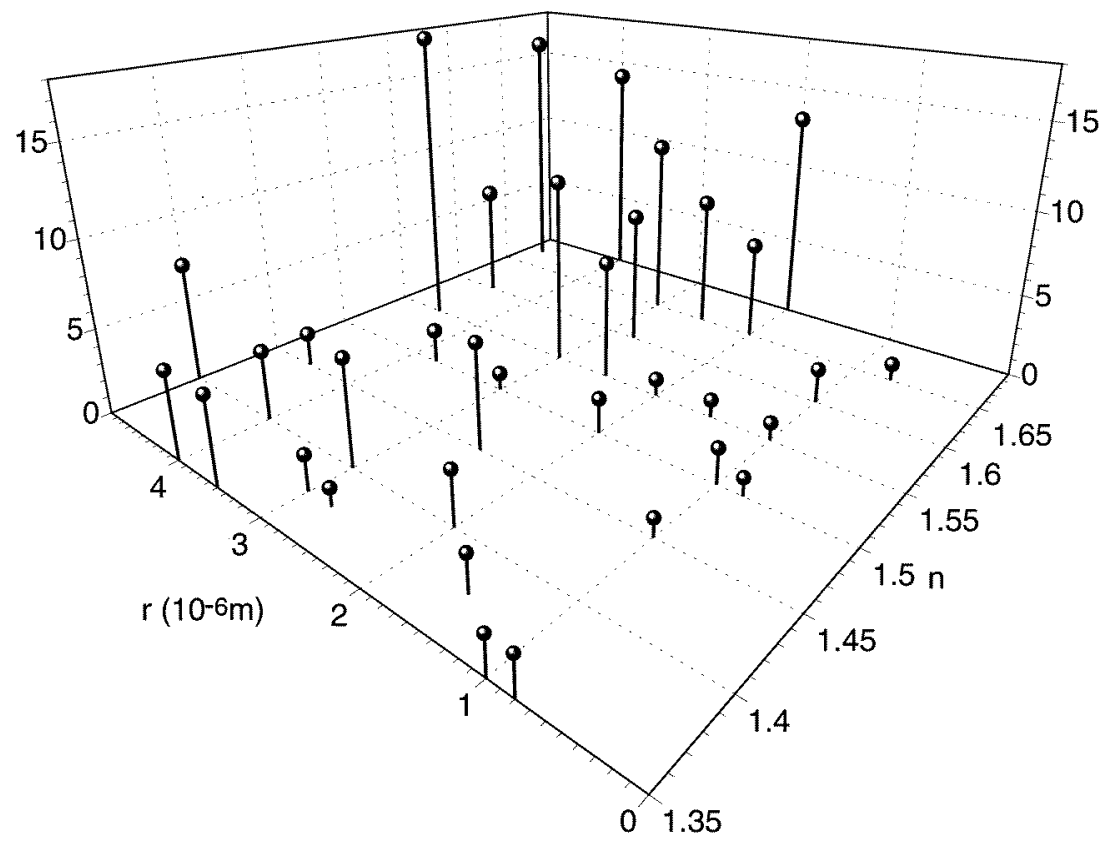

Figure 2. Numbers of local minima for random locations of the global solution point on the $(r, n)$ plane.

generate the data, and is given by

$$
n^{*}-0.01 \leqslant n \leqslant n^{*}+0.01 \quad \text { and } \quad r^{*}-0.075 \leqslant r \leqslant r^{*}+0.075 .
$$

The data from table 8 together with additional points are graphically presented in figure 2 . It can be seen that whilst the number of minima varies widely, it generally remains low for radii of less than $2 \mu \mathrm{m}$ and for refractive indices of less than 1.55 .

Although the number of points examined is small, the general character of the observed trend is probably quite universal. The fact that the number of local minima is large when both $r$ and $n$ are large is most probably related to properties of the scattered intensity itself, demonstrated, for example, by its angular dependence. It is a well known general feature of scattering on particles comparable in size with the wavelength of light that the number of peaks present in the angular representation of the scattered intensity is roughly proportional to both the size of the particle and its refractive index (the latter with respect to the surrounding medium). Therefore, the error functions (1) and (2) can be expected to have more minima for larger values of these parameters. These observations on the behaviour of the error function (2) should be helpful in future work, especially when dealing with experimental data.

The experiments reported so far have used a rather small box around the nominal global solution (the point $\left(n^{*}, r^{*}\right)$ ). Even so, in some cases a large number of local solutions was found. The area around the solution is now increased to give boxes such that

$$
n^{*}-0.05 \leqslant n \leqslant n^{*}+0.05 \quad \text { and } \quad r^{*}-0.15 \leqslant r \leqslant r^{*}+0.15 .
$$

The results are shown in table 9 , which is of the same form as table 8 except that the expected number of minima given by (7) is also shown. 
Table 9. Results of minimizing (2) with respect to $n$ and $r$ in boxes of size $0.1 \times 0.3$.

\begin{tabular}{|c|c|c|c|c|c|}
\hline \multirow[b]{2}{*}{ No } & \multicolumn{2}{|c|}{ nominal point } & \multirow[b]{2}{*}{ Iterations } & \multirow[b]{2}{*}{ Minima } & \multirow[b]{2}{*}{ Expected } \\
\hline & Ref. index & Radius $(\mu \mathrm{m})$ & & & \\
\hline 1 & 1.350 & 3.500 & 274 & 20 & 22 \\
\hline 2 & 1.350 & 4.000 & 209 & 19 & 22 \\
\hline 3 & 1.375 & 3.000 & 303 & 22 & 24 \\
\hline 4 & 1.400 & 3.000 & 375 & 18 & 19 \\
\hline 5 & 1.500 & 2.000 & 179 & 12 & 13 \\
\hline 6 & 1.500 & 3.750 & 267 & 31 & 36 \\
\hline 7 & 1.550 & 3.000 & 393 & 45 & 51 \\
\hline 8 & 1.550 & 4.500 & 250 & 52 & 66 \\
\hline 9 & 1.600 & 4.500 & 399 & 47 & 53 \\
\hline 10 & 1.650 & 2.500 & 379 & 44 & 50 \\
\hline
\end{tabular}

Table 10. Computational time used for minimizing (2) with respect to $n$ and $r$ using two different methods. Numbers in brackets give the number of minima found by the clustering method.

\begin{tabular}{ccrcc}
\hline \multicolumn{2}{c}{ Starting point } & & \multicolumn{2}{c}{ Time used } \\
\cline { 5 - 5 } \cline { 5 - 5 } Ref. index & \multirow{2}{*}{ Radius $(\mu \mathrm{m})$} & & Deep's & Clustering \\
\hline 1.450 & 4.500 & & 9.86 & $512.2(2)$ \\
1.500 & 0.800 & & 7.26 & $79.1(1)$ \\
1.550 & 3.000 & & 10.52 & $5011.5(11)$ \\
1.600 & 4.500 & & 16.04 & $2773.6(7)$ \\
\hline
\end{tabular}

In every case the number of local minima has increased considerably, compared with table 8. Because of the number of minima, many iterations of the clustering algorithm would be required to find all the local solutions. In the results given in table 8 there were 18 local minima and 726 optimizations were needed, so the number of iterations for, say, 50 minima could be expected to be more than 3000 . Therefore, the algorithm was not allowed to run until the end. As in table 8 and figure 2, there are fewer local minima for refractive indices smaller than 1.55 .

Finally, typical computation times for the two global methods under study are shown in table 10. It can be seen that, on average, the clustering approach is about 200 times slower than Deep's random search.

\section{Conclusions}

Two different global optimization methods were used for solving an inverse scattering problem. Deep's method seeks only one minimum in the region of interest. Although this method is much faster, the clustering method appears to be more reliable and it gives a clearer picture of the behaviour of the error function in terms of both the number of local minima and the values of the error function. These features may be particularly valuable when dealing with experimental data. In this case the error function cannot be expected to be zero at the global solution because of measurement inaccuracy and noise. Moreover, for the same reason, the solution with the smallest error may not be the correct one [18]. On the other hand, since Deep's method is faster it could be used for initial search in certain 
areas of the solution space, followed by the application of the clustering approach for a more thorough analysis in the neighbourhood of the newly found solutions.

The results presented here clearly show that the error function (2) has many local minima. When dealing with a small region around the global solution (i.e. for the results shown in table 8) the situation is not too critical, as in all 15 cases examined here all local solutions were found (with the probability of 95\%). However, when the region is increased in size, the efficiency of the method quickly deteriorates. It is only when a reasonably small box around the solution can be used that the clustering algorithm becomes a practical method for seeking the global minimum of function (2). This shows the need for a good initial estimate of the particle radius and refractive index, which may be particularly important when dealing with experimental measurements of scattered light. The initial guess may be provided, for example, by looking at peak positions in the experimental scattering pattern [3, 8, 25-27].

While the number of minima found may vary with the size of the initial sample, it is usually the higher-valued local minima that are overlooked when the number of sample points is reduced. This gives a reason for believing that the computational costs of the clustering algorithm can be reduced when it is applied to the inverse light-scattering problem in practice. However, this matter needs further investigation, because some local minima are very close to each other, and so the critical distance $r_{k}$ must be small in order to distinguish between two very close minima. This will tend to increase the cost of the clustering algorithm because it will increase the number of points that are kept in the reduced sample.

We conclude by making some general comments on the behaviour of the clustering algorithm. First, because of the local phase it contains, the algorithm searches only for local minima in the given region. Therefore if a function reaches its optimum on the boundary of the region, the algorithm would not declare it as a minimum, as it only looks for stationary points. This is not a serious drawback, however, because the boundaries are artificially implemented, so they can always be extended in order to obtain the optimum.

I was also told that the algorithm might not always find all local solutions. This fact could be disregarded since, in order to accelerate the convergence, the algorithm intentionally avoids local minima with relatively high function values. Alternatively, the initial sample could be increased. In all tests done so far, the optimal solution (that with the zero error) was among the local minima found, irrespective of sample size.

Rinnooy Kan and Timmer [15] gave some computational results for their algorithm. The results they provide are somewhat worse than those reported here, in the sense that they include cases when a local minimum has been declared as the global one. One of the reasons they give for this is that the regions of attraction of local minima may contain sample points whose function values are smaller than the smallest function value at a sample point in the region of attraction of the global minimum. Another, simpler, explanation is that, in one of their examples, the global minimum was close to other minima.

It has also been noted that, if the number of local minima is large, the stopping rule (8) is not reasonable. In particular, the rule is criticized because it tests the probability of finding all local minima, not the global minimum [22].

If there is no need to find all minima, a better stopping rule would be to terminate the algorithm if the total relative volume of the observed regions of attractions exceeds a prescribed value $t(0<t<1)$ [28]:

$$
\frac{(N-W-1)(N+W)}{N(N-1)} \geqslant t .
$$

For example, for case 6 from table 9, where 31 minima were found after 267 local 
searches, expression (9) yields an estimate that approximately $78 \%$ of the region of interest has been searched. Boender and Rinnooy Kan [28] also suggested that there are optimal Bayesian stopping rules which weight the cost and potential benefits of further experiments against each other.

In conclusion, optimization methods offer a potentially useful approach to solving inverse light scattering problems for small particles. The difficulty arising from the presence of numerous local solutions can be overcome by the use of stochastic global optimization methods, particularly the clustering approach. In the initial stages, however, the clustering method could be assisted by faster techniques, such as Deep's random search and/or a good initial estimate of the radius and refractive index of the particle. The simple problem examined here-the case of a homogeneous, non-absorbing sphere-was a two-parameter one. However, extending the method investigated to more general cases, with larger numbers of parameters (such as layered spheres or similar inhomogeneous particles, size distributions, etc), should not present major difficulties as long as solutions to the direct problem are available and the number of parameters is small. Neither is it required to provide scattering data extending from $0^{\circ}$ to $180^{\circ}$-recent research has shown that relatively narrow scattering angle ranges (a few tens of degrees) and sampling intervals only just avoiding aliasing are sufficient for particle radius and refractive index recovery in a context similar to the one discussed here [10].

\section{References}

[1] de Pieri L A, Ludlow I K and Waites W M 1993 The application of laser diffractometry to study the water content of spores of Bacillus sphaericus with different heat resistances J. Appl. Bacteriol. 74 578-82

[2] Ulanowski Z and Ludlow I K 1989 Water distribution, size and wall thickness in lycoperdon pyriforme spores Mycolog. Res. 93 28-32

[3] Ulanowski Z 1988 Investigations of microbial physiology and cell structure using laser diffractometry $P h D$ Thesis Hatfield Polytechnic, Hatfield

[4] Wyatt P J 1980 Some chemical, physical and optical properties of fly ash particles Appl. Opt. 7 975-83

[5] Bayvel L P and Jones A R 1981 Electromagnetic Scattering and its Applications (London: Applied Science)

[6] Bohren C F and Huffman D R 1983 Absorption and Scattering of Light by Small Particles (New York: Wiley)

[7] Jones M R, Curry B P, Brewster M Q and Leong K H 1994 Inversion of light-scattering measurements for particle size and optical constants: theoretical study Appl. Opt. 33 4025-34

[8] Maltsev V P and Lopatin V N 1997 Parametric solution of the inverse light-scattering problem for individual spherical particles Appl. Opt. 36 6102-8

[9] Quist G M and Wyatt P J 1985 Empirical solution to the inverse light scattering problem by the optical strip-map technique J. Opt. Soc. Am. A 2 1979-85

[10] Ulanowski Z, Wang Z, Kaye P H and Ludlow I K 1998 Application of neural networks to the inverse light scattering problem for spheres Appl. Opt. 37 4027-33

[11] Ludlow I K and Everitt J 1995 The application of Gegenbauer analysis to light scattering from spheres: theory Phys. Rev. E 51 2516-26

[12] Ludlow I K and Everitt J 1996 Systematic behaviour of the Mie scattering coefficients of spheres as a function of order Phys. Rev. E 53 2909-24

[13] Deep K and Evans D J 1994 A parallel random search global optimization method Technical Report 882, Computer Studies, Loughborough University of Technology

[14] Rinnooy Kan A H G and Timmer G T 1987 Stochastic global optimisation methods, part I: clustering methods Math. Program. 39 27-56

[15] Rinnooy Kan A H G and Timmer G T 1987 Stochastic global optimisation methods, part II: multilevel methods Math. Program. 39 57-78

[16] Bartholomew-Biggs M C, Ulanowski Z and Zakovic S 1994 A parameter estimation problem with multiple solutions arising in laser diffractometry Technical Report 281, NOC, University of Hertfordshire, Hatfield

[17] Bartholomew-Biggs M C, Ulanowski Z and Zakovic S 1994 Further experience with least squares solutions of an inverse light scattering problem Technical Report 294, NOC, University of Hertfordshire, Hatfield 
[18] Zakovic S 1997 Global optimization applied to an inverse light scattering problem PhD Thesis University of Hertfordshire, Hatfield

[19] Mireles R 1966 The inverse problem of electromagnetic theory I. Uniqueness theorem for cylinders J. Math. Phys. 45 179-85

[20] Zakovic S, Ulanowski Z and Bartholomew-Biggs M C 1997 Application of global optimisation to particle identification using light scattering Technical Report 325, NOC, University of Hertfordshire, Hatfield

[21] Shanker (Deep) K 1988 Computational algorithms for global optimization problems and their applications with particular reference to geophysics $P h D$ Thesis University of Roorkee, Roorkee

[22] Dixon L C W and Jha M 1993 Parallel algorithms for global optimisation J. Opt. Theor. Appl. 79 385-95

[23] Boender C G E 1984 The generalized multinomial distribution: a Bayesian analysis and applications PhD Thesis Erasmus University, Rotterdam

[24] Numerical Optimization Centre 1989 OPTIMA Manual 8th edn University of Hertfordshire, Hatfield

[25] Maron S H and Elder M E 1963 Determination of latex particle size by light scattering J. Colloid Sci. 18 $107-18$

[26] Kerker M 1969 The Scattering of Light (London: Academic) pp 344-9

[27] Robillard F and Patitsas A J 1974 Determination of size, size distribution and refractive index of Dow latexes ep-1358-38 by the Mie scattering method Can. J. Phys. 52 1571-82

[28] Boender C G E and Rinnooy Kan A H G 1987 Bayesian stopping rules for multistart global optimisation methods Math. Program. 37 59-80 